\title{
Parameter analysis of numerical simulation on flow around a cylinder
}

\author{
YOU Yi, , a , *YAN Zhitao, ${ }^{3,1, b}$, ZHONG Yongli ${ }^{1, \mathrm{c}}$ and ZHANG Long ${ }^{2}$ \\ ${ }^{1}$ School of Civil Engineering, Chongqing University, Chongqing, China \\ ${ }^{2}$ State Grid Xinjiang Electric Power Company Electric Power Research Institute, Urumqi, China \\ ${ }^{3}$ School of Civil Engineering, Chongqing University of Science and technology, Chongqing, China \\ aemail:97080463@qq.com, bemail: yanzhitao@cqu.edu.cn, cemail: zhongyongli@cqu.edu.cn
}

\begin{abstract}
Keywords: large eddy simulation; flow around cylinder; parameter analysis.
Abstract. The parametric analysis is carried out by using numerical simulation methods for the flow around a single cylinder. The influence of the three-dimensional effect, grid form, time step, turbulence model and sub-grid scale models on the calculation results are discussed, respectively. The analysis results show that the numerical simulation of the flow around a single cylinder cannot ignore the influence of the three-dimensional effect. The grid form of the core region and the grid quality of the near-wall have a great influence on the calculation results When time step is less than $0.001 \mathrm{~s}$, the calculated value of drag coefficient is more close to experimental results. The Smagorinsky-Lilly model of the large eddy simulation is appropriate for the analysis of flow around the cylinder.
\end{abstract}

\section{Introduction}

In the field of fluid mechanics, the flow around a cylinder is a very classical problem. There are lots of researches about this problem ${ }^{[1,2]}$, which include theoretical analysis, experimental research and numerical simulation ${ }^{[3-5]}$. There are many factors that affect the accuracy of the calculation results, such as the size of the computational domain, three-dimensional effect, grid form, grid quality, time step and turbulence model. The experimental research on the problem of flow around a single cylinder has been developed maturely, and many scholars have given a lot of valuable experimental data. Cantwell and Coles ${ }^{[6]}$ carried out a test on flow around a smooth single cylinder with the Reynolds number $\mathrm{Re}=1.4 \times 10^{5}$ in 1983 , which provided the detailed data of the load characteristics and flow field distribution on the cylindrical surface. In this paper, a parameter analysis of numerical simulation on the flow around a single cylinder is carried out to obtain the reasonable calculation parameters and ensure the correctness and accuracy of the numerical simulation method.

\section{Calculation Model and Parameters}

The setting of the computational domain is shown in Fig.1. Where, D represents the diameter of the cylinder $(\mathrm{D}=57 \mathrm{~mm})$. The position of the coordinate origin is at the center of the bottom of the cylinder. The direction of the $\mathrm{X}, \mathrm{Y}$ and $\mathrm{Z}$ axis of the coordinate system are parallel to the along-wind, across-wind, and the height direction, respectively. The flow runs from the left to the right, the entry boundary is the velocity inlet and the outlet is a pressure outlet, the calculated wind velocity $\mathrm{V}=1.84 \mathrm{~m} / \mathrm{s}$. The upper and lower boundaries are symmetric boundaries, and the left and right boundaries also are symmetric boundaries. The no-slip boundary conditions were used at cylinder surface.

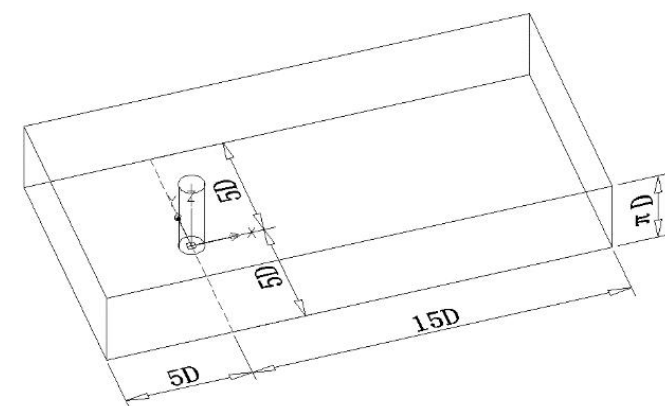

Fig. 1 Computational domain

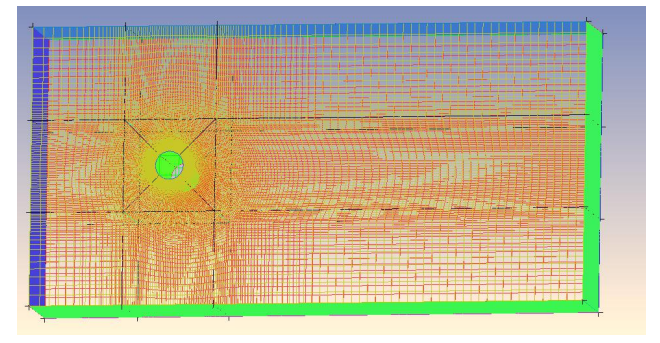

Fig. 2 Mesh of the computational domain 
The ICEM software was applied to mesh the flow field. Since the complexity of flow field near the cylinder, it is necessary to densify the near-wall grid, as shown in Fig.2. The second-order upwind difference scheme was adopted for the convection term, and the SIMPLE algorithm was used to solve the pressure-velocity coupling equation. The settings for other comparison parameters are shown in Table 1. Where, pattern 1, pattern 2 and pattern 3 represent the Smagorinsky-Lilly, dynamic Smagorinsky-Lilly and SST k- $\omega$ model, respectively.

Tab 1 Settings of computational conditions

\begin{tabular}{ccccc}
\hline Case & Grid in core region & Boundary layer $\left(y^{+}\right)$ & Time step $(s)$ & Pattern \\
\hline \multicolumn{5}{c}{ Group A:three-dimensional effect } \\
\hline A1 & $120 \times 42 \times 1$ & $0.006 \mathrm{~mm}(0.7)$ & $5 \times 10^{-4}$ & Pattern 1 \\
A2 & $120 \times 42 \times 32$ & $0.006 \mathrm{~mm}(0.7)$ & $5 \times 10^{-4}$ & Pattern 1 \\
\hline \multicolumn{5}{c}{ Group B: boundary layer and grid } \\
\hline B1 & Unstructured grid & $0.006 \mathrm{~mm}(0.7)$ & $5 \times 10^{-4}$ & Pattern 1 \\
B2 & $120 \times 42 \times 32$ & $0.006 \mathrm{~mm}(0.7)$ & $5 \times 10^{-4}$ & Pattern 1 \\
B3 & $80 \times 42 \times 32$ & $0.08 \mathrm{~mm}(10)$ & $5 \times 10^{-4}$ & Pattern 1 \\
\hline \multicolumn{5}{c}{ Group B: time step } \\
C1 & $120 \times 42 \times 32$ & $0.006 \mathrm{~mm}(0.7)$ & $1 \times 10^{-3}$ & Pattern 1 \\
C2 & $120 \times 42 \times 32$ & $0.006 \mathrm{~mm}(0.7)$ & $5 \times 10^{-4}$ & Pattern 1 \\
C2 & $120 \times 42 \times 32$ & $0.006 \mathrm{~mm}(0.7)$ & $1 \times 10^{-4}$ & Pattern 1 \\
\hline \multicolumn{5}{c}{$0.006 \mathrm{~mm}(0.7)$} \\
D1 & Group D: turbulence model and sub-grid scale models & $5 \times 10^{-4}$ & Pattern 1 \\
D2 & $120 \times 42 \times 32$ & $0.006 \mathrm{~mm}(0.7)$ & $5 \times 10^{-4}$ & Pattern 2 \\
D3 & $120 \times 42 \times 32$ & $0.006 \mathrm{~mm}(0.7)$ & $5 \times 10^{-4}$ & Pattern 3 \\
\hline
\end{tabular}

\section{Results and Discussion}

By comparing the simulation cases of the above four groups, the influence of the three-dimensional effect, the grid form, the time step, the turbulence model and the sub-grid scale models of large eddy simulation are discussed, the calculation results are shown in Table 2.

Tab 2 The results of different computational conditions

\begin{tabular}{cccc}
\hline & Case & $C_{D}$ & $S_{t}$ \\
\hline & $\mathrm{A} 1$ & 0.85 & 0.272 \\
$\mathrm{~A} 2$ & 1.28 & 0.214 \\
$\mathrm{~B} 1$ & 1.14 & 0.211 \\
& $\mathrm{~B} 2=\mathrm{A} 2$ & 1.28 & 0.214 \\
& $\mathrm{~B} 3$ & 0.75 & 0.251 \\
& $\mathrm{C} 1$ & 1.36 & 0.272 \\
& $\mathrm{C} 2=\mathrm{A} 2$ & 1.28 & 0.214 \\
& $\mathrm{C} 3$ & 1.21 & 0.218 \\
& $\mathrm{D} 1=\mathrm{A} 2$ & 1.28 & 0.214 \\
& $\mathrm{D} 2$ & 1.29 & 0.205 \\
Simulation & 0.81 & 0.237 \\
& D3 & 1.40 & 0.184 \\
& Literature[7] & 1.286 & 0.202 \\
& Literature[8] & 1.237 & 0.179 \\
\hline
\end{tabular}




\section{The influence of three-dimensional effect}

Studies ${ }^{[9]}$ indicated that the three-dimensional effect of the flow around the cylinder is obvious with $\mathrm{Re} \geqslant 250$. In order to study the influence of three-dimensional effect on the calculation results under high Reynolds number, the calculation result of case A1 and A2 are compared. Taking into account other factors that may cause interference, the same grid form, time step and the sub-grid scale models were adopted for these two cases. The difference between the above two cases is that the model corresponding to case A1 only discretized by a layer grid in the direction of the $\mathrm{Z}$ axis, which is used to simulate the two-dimensional condition. However, the model corresponding to case A2 discretized by 32 layers grid in the direction of the $Z$ axis, which is used to simulate the three-dimensional condition.

It can be seen from the table 2 that the calculated results of case A1 are quite different from that of the simulation ${ }^{[7-8]}$ and experimental results ${ }^{[6]}$. Among them, the drag coefficient is $31.3 \%$ smaller than that of experimental results, while the Strouhal number $(\mathrm{St})$ is $52 \%$ larger than that of experimental results. The calculated results of the case A2 are in good agreement with that of the numerical simulation and the experimental results.

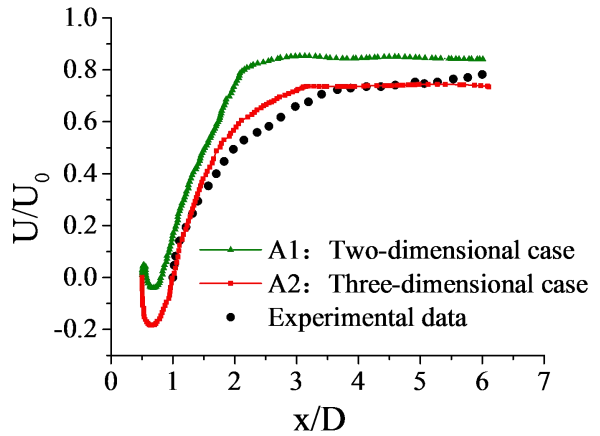

(a) Time-averaged velocity distribution

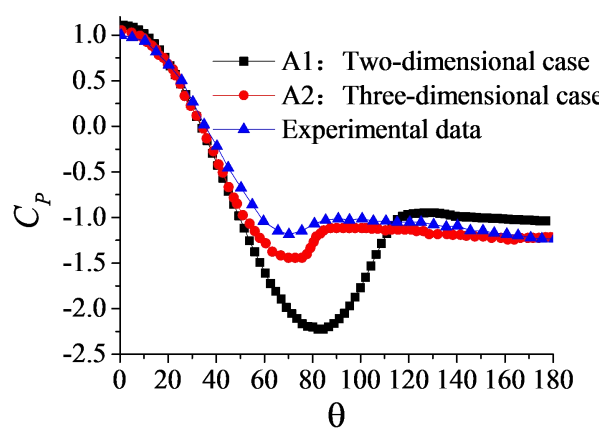

(b) Steady-state pressure coefficient distribution

Fig. 3 The influence of three-dimensional effect

Fig.3 (a) shows the time-averaged velocity distribution of the flow field in $\mathrm{X}$ axis direction calculated by case A1 and A2. It can be seen from the Fig.3 (a) that the length of recirculation zone of the two-dimensional condition in near wake region is very small, which is quite different from the experimental results. The calculated results of the three-dimensional condition agree well with the experimental results, and the length of the recirculation zone is approximately equal to the diameter of the cylinder. Fig.3 (b) shows the distribution of steady-state pressure coefficient along the circumferential direction calculated by case A1 and A2. It can be seen from the Fig.3 (b) that in the two-dimensional numerical simulation, the distribution of steady-state pressure coefficient on the cylindrical surface is obviously different from that of experimental results.

In order to intuitively observe the three-dimensional effect of flow around a cylinder, the streamline pattern around the cylinder at 4 different heights in a given time are captured in Fig.4. It can be clearly seen that the flow field characteristics behind the cylinder are obviously different from each other, which indicates that there exists a three-dimensional effect in the subcritical region $\left(\mathrm{Re}=1.4 \times 10^{5}\right)$.
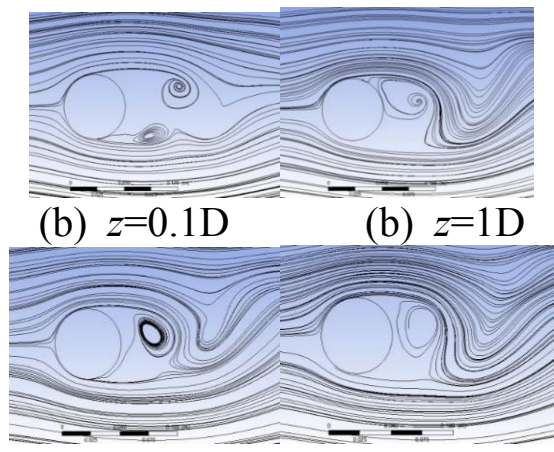

$\begin{array}{ll}\text { (c) } z=2 \mathrm{D} & \text { (d) } z=3 \mathrm{D}\end{array}$

Fig. 4 The streamline pattern at different heights on the cross section 
The above analysis shows that the three-dimensional effect of the flow around the cylinder is obvious, and the errors obtained from the two-dimensional large eddy simulation are very large. Therefore, it is necessary to consider the three-dimensional effect of the flow around a cylinder with high Reynolds number.

\section{The influence of grid conditions}

It can be seen from the table 2 that the meshing method and the quality of near-wall grid have an effect on the numerical simulation results. The flow field of case B2 is discretized by structured grids, and the quality of the near-wall grid also well controlled. The obtained results are the closest to the experimental data. Although the flow field of case B3 is also discretized by structured grids, the grids in core and near-wall regions are not dense enough, which results in a $38.6 \%$ error between the calculated results and the experimental data. While the flow field of case B1 is discretized by the unstructured grids in core region, it still obtains a better calculation result under the condition of ensuring the high quality of grids in core and near-wall region. The $\mathrm{C}_{\mathrm{D}}$ coefficient is only $7.8 \%$ smaller than that of case B2.
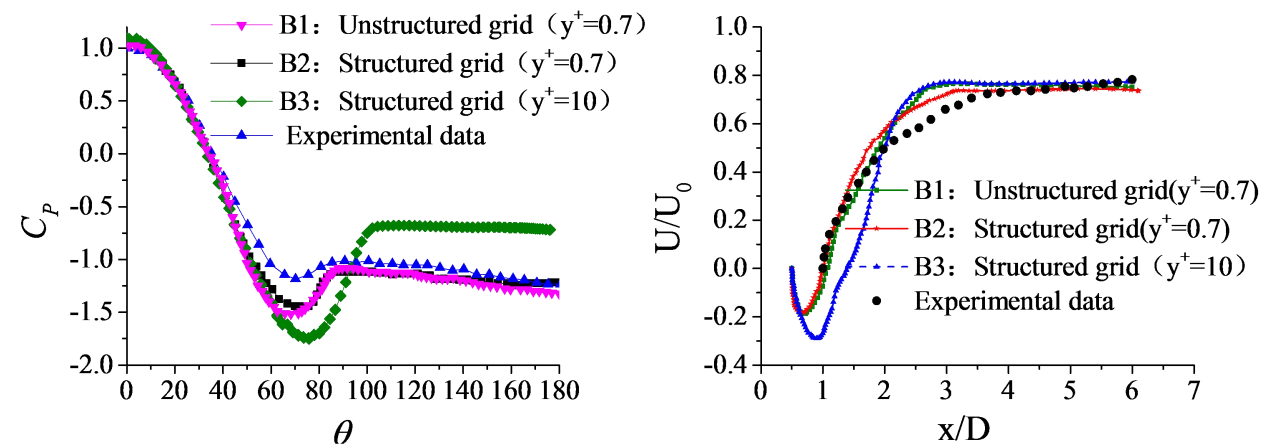

(a) Steady-state pressure coefficient distribution (b) Time-averaged velocity distribution

Fig. 5 The influence of meshing conditions

The influence of grid conditions on the distribution of steady-state pressure coefficient and time-averaged velocity are shown in Fig. 5. Both the flow field of case B2 and B3 are discretized by structured grids, but the steady-state pressure coefficient distribution and the flow field characteristics of the wake region are quite different from each other. This is due to the difference of the grid number and the grid quality of the near-wall region in the above two models. Fig. 5 (a) shows that when the grids in near-wall regions are not dense enough $(\mathrm{y}+=10)$, the negative pressure on the side of the cylinder is too large, and compared with the experimental results, the maximum negative pressure position moves backward. Fig.5 (b) shows that the length of recirculation zone and maximum reflux velocity of case B3 is too large, which is not in good agreement with the experimental results. From the comparison of case B1 and B2, it can be seen that the flow field discretized by unstructured grids can also obtain a better simulation result while the grids in near-wall regions are dense enough.

As a result, it can be seen that the grid conditions have great influence on the results of large eddy simulation. The large eddy simulation method can well simulate the problem of flow around the cylinder under the condition of improving the grid quality in core and near-wall region.

\section{The influence of time step}

In order to analyze the influence of time step on the calculation results, the time step $0.001 \mathrm{~s}$, $0.0001 \mathrm{~s}, 0.0005 \mathrm{~s}$ are discussed, respectively. It can be seen from table 2 that the calculation results of the above three cases are close to each other, and the shorter the time step, the closer the calculated value of the drag coefficient to the experimental result. 

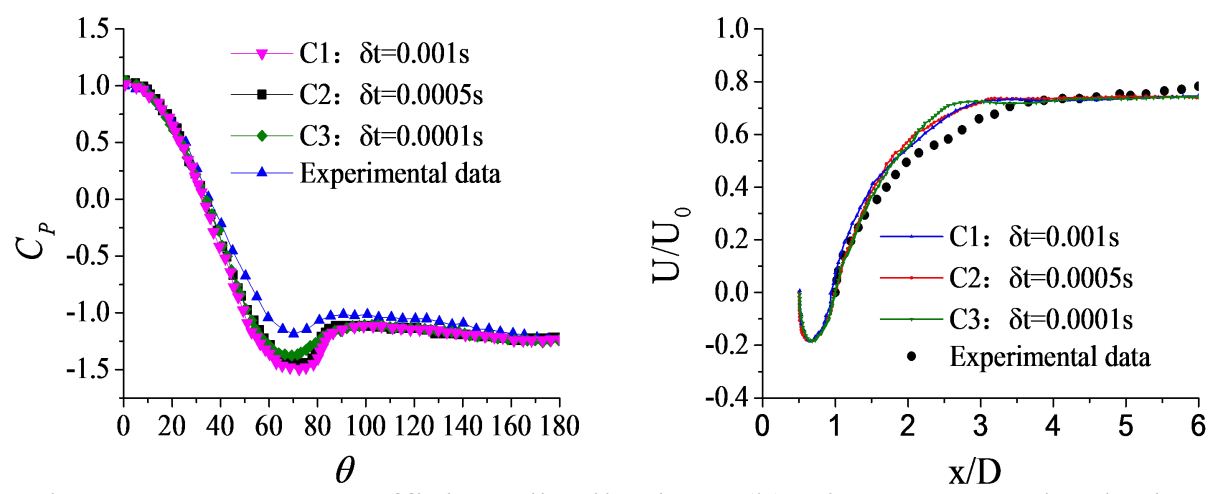

(a) Steady-state pressure coefficient distribution (b) Time-averaged velocity distribution

Fig. 6 The influence of computational time step

Fig.6 (a) shows the distribution of steady-state pressure coefficient along circumferential direction at the height of $\mathrm{z} / \mathrm{D}=0.5 \pi$ under the condition of the above three kinds of time step. As can be seen from Fig.6 (a), with the decrease of time step, the numerical results are closer to the experimental results. Fig.6 (b) shows the time-averaged velocity distribution of the flow field in $\mathrm{X}$ axis direction under the condition of the above three kinds of time step. The results of the above three cases agree well with the experimental results. It indicates that the time step has little effect on the distribution of pressure and flow field when the time step is controlled within $0.001 \mathrm{~s}$. However, the time step has great effect on computation time, with the decrease of time step, the computation time will increase greatly. Based on the above considerations, the time step is set up to $0.0005 \mathrm{~s}$, which can get a better numerical simulation result.

\section{The influence of turbulence model and sub-grid scale models}

In order to analyze the influence of turbulence model and sub-grid scale models on the flow around cylinder at a high Reynolds number, the Smagorinsky-Lilly model, dynamic Smagorinsky-Lilly model and SST k- $\omega$ model are discussed. It can be seen from table 2 that the results of large eddy simulation are in good agreement with the experimental results. However, the error of drag coefficient calculated by SST k- $\omega$ model is large, which reaches $34.5 \%$.
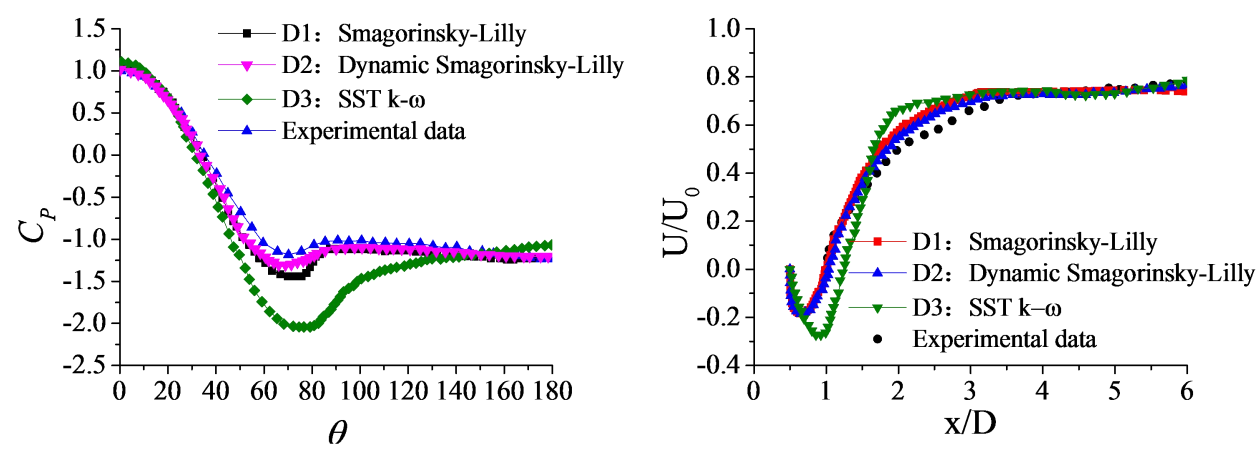

(a) Steady-state pressure coefficient distribution (b) Time-averaged velocity distribution

Fig. 7 The influence of turbulence models and sub-grid scale models

Fig. 7 shows the effects of turbulence models on the distribution of steady-state pressure coefficient and time-averaged velocity. There is a larger difference between the distribution of steady-state pressure coefficient or the distribution of time-averaged velocity calculated by case D1 and D3. As can be seen from Fig.7 (a), the negative pressure on the side of the cylinder calculated by the case D3 is too large. Compared with the experimental results, the location of the maximum negative pressure on the cylinder moves backward. Fig.7 (b) shows that the length of recirculation zone and the maximum reflux velocity calculated by the case D3 are larger than that of experimental results. From the calculation results of case D1 and D2, the sub-grid scale models have little effect on the calculation results, and both of the calculation results agree well with the experimental results. However, the amount of calculation required by the dynamic Smagorinsky-Lilly model is much 
larger than that of the Smagorinsky-Lilly model. Therefore, the Smagorinsky-Lilly model of the large eddy simulation is more appropriate for the analysis of flow around the cylinder.

\section{Conclusion}

The parametric analysis is carried out by using numerical simulation methods for the flow around a single cylinder. The influence of the three-dimensional effect, the grid form, the time step, the turbulence model and the sub-grid scale models on the calculation results are discussed. The main conclusions are as follows:

(1) The numerical simulation of the flow around a single cylinder cannot ignore the influence of the three-dimensional effect. The two-dimensional model of large eddy simulation cannot obtain accurate wake characteristics and the distribution of steady-state pressure coefficient on the surface of cylinder, which leads to a larger error in the calculation of CD and St.

(2) The grid form has a great influence on the calculation results, especially the near-wall grid. In order to obtain a better numerical simulation result, the $\mathrm{y}^{+}$should be controlled within 1 . The calculation results of the core region discretized by structured grids are better than that discretized by unstructured grids. Compared with the experimental results, the results of core region discretized by unstructured grids are smaller, and the error can be controlled within $10 \%$.

(3) When value of the time step is a smaller one ( $\delta \mathrm{t} \leqslant 0.001 \mathrm{~s})$, the time step has little effect on the results. The calculated flow field characteristics and pressure distribution are basically the same at different time steps.

(4) Compared with the reynolds-averaged navier-stokes simulation, the large eddy simulation can more fully present the continuous motion and development of the vortex in wake, and the simulation results are closer to the experimental data. The Smagorinsky-Lilly model of the large eddy simulation is more appropriate for the analysis of flow around the cylinder.

\section{Acknowledgements}

This work was financially supported by the National Natural Science Foundation of China (Grant No. 51478069).

\section{References}

[1] EAchenbach. Distribution of local pressure and skin friction around a circular cylinder in cross-flow up to $\mathrm{Re}=5 \times 106$ [J]. Journal of Fluid Mechanics, 1968, 34(4):625-639.

[2] P. Beaudan and P. Moin. Numerical experiments on the flow past a circular cylinder at sub-critical Reynolds number[R]. Stanford, California: Department of Mechanical Engineering, Stanford Univer-sity, 1994.

[3] C. H. K. Williamson. Vortex dynamics in the cylinder wake[J]. Annual Review of Fluid Mechanics, 1996, 28:477-539.

[4] M. M. Zdravkovich. Flow around circular cylinders; volume 1. fundamentals[M]. Oxford: Oxford University Press, 1997.

[5] M. M. Zdravkovich. Flow around circular cylinders, Vol. 2: Applications[M]. Oxford: Oxford Uni-versity Press, 2003.

[6] Cantwell B, Coles D. An experimental study of entrainment and transport in the turbulent near wake of a circular cylinder [J]. Journal of Fluid Mechanics, 1983, 139: 321-374.

[7] Tutar M, Holdob A E. Computational modeling of flow around a circular cylinder in sub-critical flow regime with various turbulence models [J]. International Journal for Numerical Methods in Fluids, 2015, 35(7): 763-784.

[8] Breuer M. A challenging test case for large eddy simulation: high Reynolds number circular cylinder flow [J]. International Journal of Heat and Fluid Flow, 2000, 21(5): 648-654.

[9] Kravchenko, A.G., Moin, P. Numerical studies of flow over a circular cylinder at ReD=3900[J]. Physics of Fluids, 2000, 12(2):403-417. 